\title{
Unusual Cause of Back Pain in an Adolescent Patient: A Case Report and Natural History of Aggressive Vertebral Hemangioma in Children
}

\author{
Bhawna Jha, MD, and Arabinda K. Choudhary, MD
}

From: Hershey Medical Center, Hershey, PA

Dr. Jha is with the Department of Neurology, Hershey Medical Center, Penn State University, Hershey PA. Dr. Choudhary is Assistant Professor of Radiology, Department of Radiology, Hershey Medical Center, Penn State University, Hershey, PA.

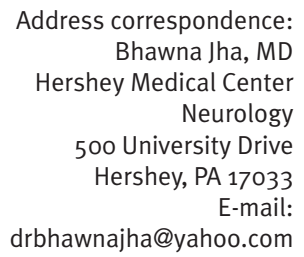
Bhawna Jha, MD Hershey Medical Center Neurology 500 University Drive Hershey, PA 17033 E-mail:

drbhawnajha@yahoo.com

Disclaimer: There was no external funding in the preparation of this manuscript.

Conflict of interest: None.

Manuscript received: 06/05/2008 Accepted for publication: 07/30/2008

Free full manuscript: www.painphysicianjournal.com
Back pain is an unusual complaint in children as compared to the adult population but is more likely to be pathological. We present a longitudinal study of an 11-year-old patient who presented with a vertebral hemangioma which remained stable for a period of about 20 months followed by aggressive growth resulting in spinal cord compression. This is only the fourth reported case of aggressive vertebral hemangioma in children; however, we document for the first time the evolution of this lesion demonstrating a period of stability followed by aggressive growth of the lesion.

We discuss the imaging differential diagnosis which includes asymptomatic vertebral hemangioma and malignant lesions. We also discuss different treatment options available and their complications. In our patient, among the risk factors for an aggressive hemangioma, female gender and involvement of posterior elements were positive. However, the natural history of this lesion is not well known in children. We need to study vertebral hemangioma further especially in children to understand the natural course and help identify lesions which have aggressive potential and prevent spinal cord compression. This will also help to manage the lesion earlier with less aggressive therapies such as embolization and ethanol injection rather than surgery.

Key words: back pain, pediatrics, vertebral hemangioma

Pain Physician 2008; 11:5:687-692
W

e present an unusual cause of back pain in an adolescent patient with an aggressive vertebral hemangioma. Only 3 cases have been reported in the literature in the pediatric age group (1-3). We demonstrate the evolution of the lesion over a period of approximately 20 months with stability followed by aggressive growth and compression of the spinal cord. There is one longitudinal follow up of aggressive vertebral hemangioma in the literature in an adolescent patient who initially presented with spinal cord compression. The parents and patient refused therapy and at 6 years follow up there was partial involution of the lesion $(2,4)$. In the absence of any other longitudinal study it is difficult to ascertain whether our case reflects a natural history or an atypical course. Low back pain in children is an uncommon complaint but is more 
likely to be pathological than in adults. As such, it should be carefully evaluated. It is important to be aware of these lesions, their natural history and management.

\section{Case Report}

An 11-year-old female presented with several year history of back pain. There was no history of trauma. There was also occasional weakness in her legs when she was tired. She had some pain in her lower lumbar area at the end of the day and sometimes with sitting for too long. However, she was in a fitness class at school where she was doing stationary bike, elliptical, and treadmill without any exacerbation of the pain. She was able to bend forward and hyperextend without any significant discomfort. There was no bowel or bladder dysfunction. Neuro- logical examination was otherwise normal with no thoracic or lumbar asymmetry. An outside MRI study was suggestive of hemangioma of T12 without any evidence of collapse or instability. The outside study was not available for review. The X-ray at presentation at our institute showed a vertebral lesion of the left body and pedicle of T12 with striations (Fig. 1A) which was consistent with known hemangioma. A CT was performed which confirmed T12 hemangioma of the majority of the left vertebral body and also part of the posterior elements (Figs. 1B \& 1C). No significant epidural component, vertebral collapse, or instability was seen. Therefore, a decision was made to follow a conservative approach with a period of immobilization with brace and close follow-up. At the 2-month follow-up, her pain was much better with wearing the brace for about 14 hours per day. No

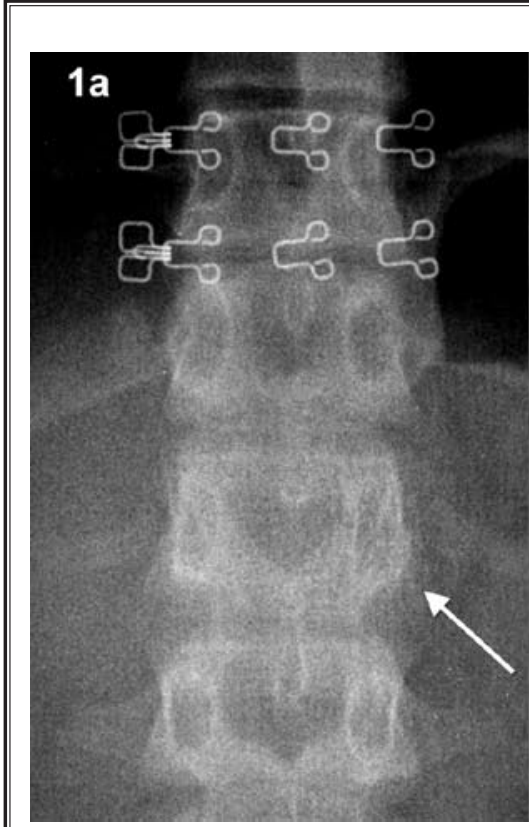

A. AP X-ray of the lumbar spine demonstrating striations and increased lucency of the left T12 vertebral body (arrow).

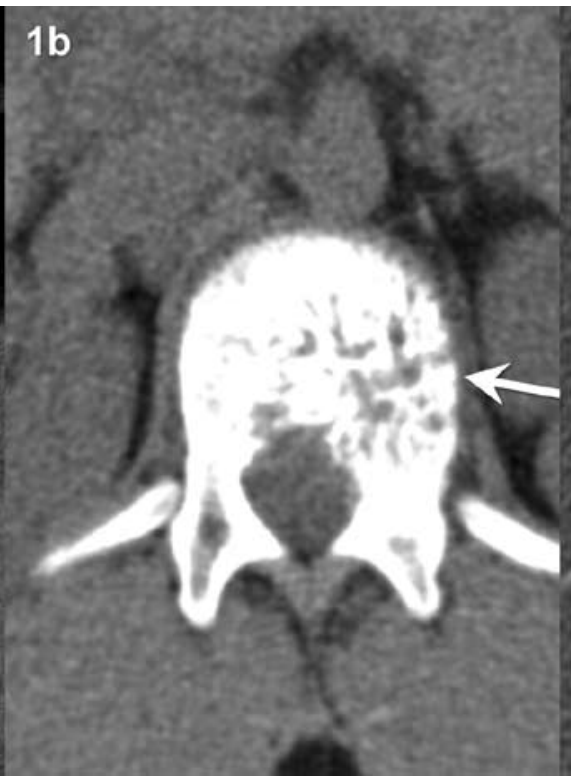

B. Axial CT image at the level of origin of superior mesenteric artery (SMA). Soft tissue window setting demonstrates abnormal hypodensity with slight expansion of left T12 vertebral body and prominent striations (arrow). No significant epidural component is seen.

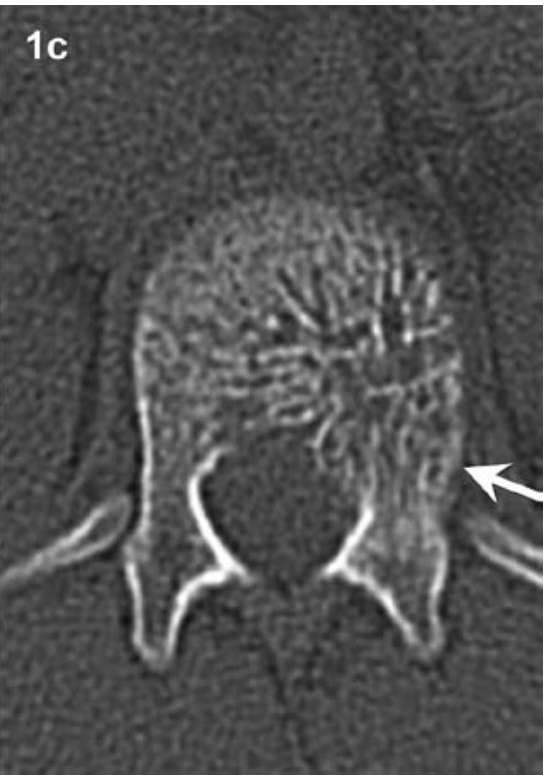

C. Axial CT image at the level of origin of superior mesenteric artery (SMA). Bony window setting demonstrates abnormal hypodensity with slight expansion of left T12 vertebral body and prominent striations. The left posterior element is also involved (arrow). No significant epidural component is seen.

Fig. 1. AP and Axial CT images of the T12 vertebral body. 
pain medication was needed. Her pain was responding well to brace treatment at a further 3 month follow-up. She was able to bend forward and hyperextend without discomfort and was pain free at the subsequent 3 months follow-up. CT did not show any significant change at this time. She was weaned out of the brace. At 6 months follow-up, she was found to be doing well with no radiographic change. A CT was repeated 5 months later due to increased back pain, and numbness and tingling in her right leg, which were new findings. The T12 hemangioma was much larger with a significant compromise of the spinal canal with an epidural soft tissue mass compressing the thecal sac (Figs. 2A \& 2B). An MRI confirmed the findings (Figs. 3A-3C). Surgery was performed with partial resection of $\mathrm{L} 1$ and fusion.

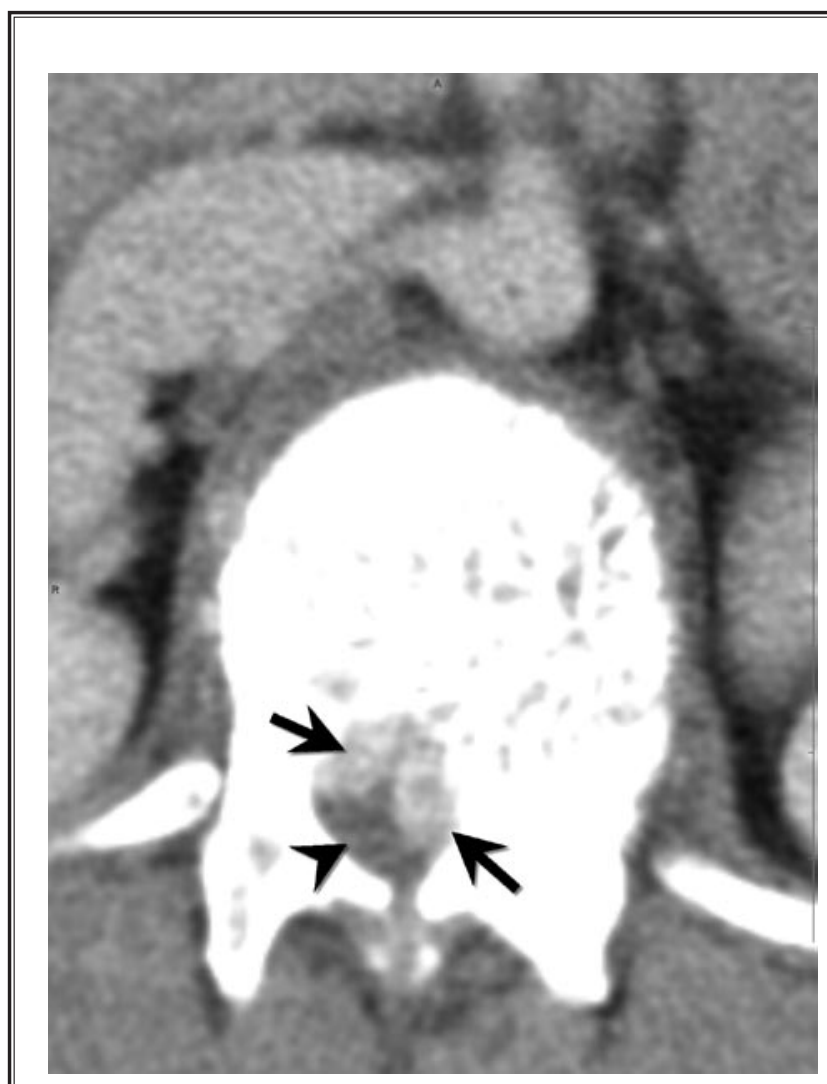

A. Post intravenous contrast medium CT image. Soft tissue window setting demonstrates a significant increase in the size of the lesion with expansion of vertebral and posterior elements as well as a large enhancing epidural soft tissue mass (arrows) compressing the thecal sac (arrowhead).

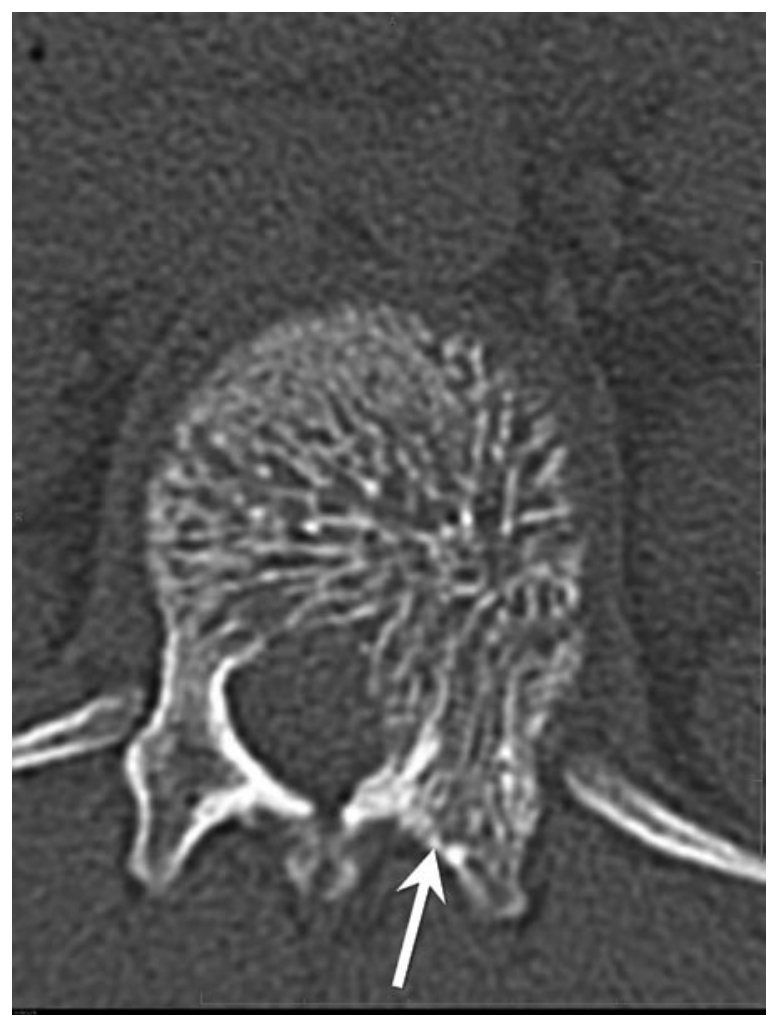

B. Axial CT image. Bony window setting demonstrates a significant increase in the size of the lesion with expansion of vertebral and posterior elements (arrow).

Fig. 2. Axial CT image at the level of origin of superior mesenteric artery (SMA). 


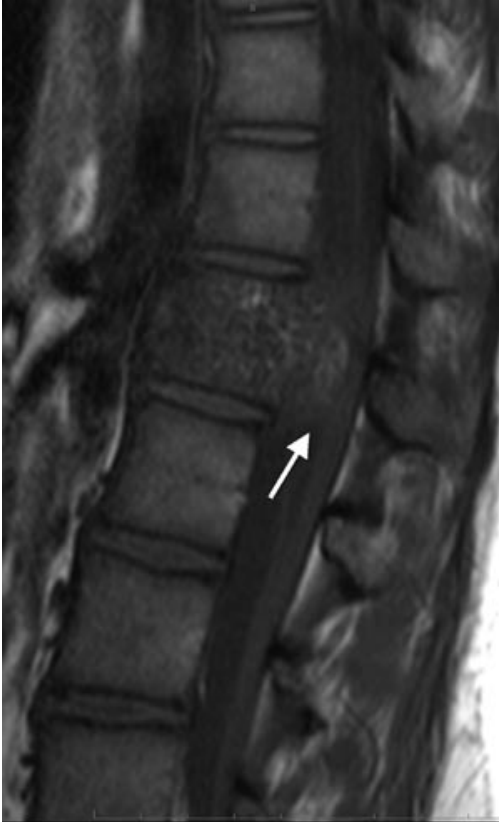

A. Sagittal MRI T1 sequence shows an expansile T12 vertebral body with diffuse hypointensity and a posterior epidural hypointense soft tissue mass (arrow) severely compressing the thecal sac.

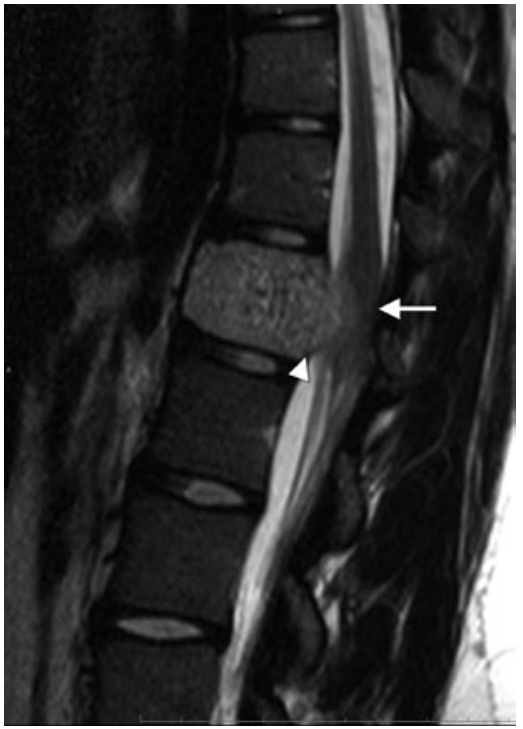

B. Sagittal MRI T2 sequence shows an expansile T12 vertebral body with diffuse hyperintensity and a posterior epidural hyperintense soft tissue mass (arrowhead) severely compressing the thecal sac (arrow).

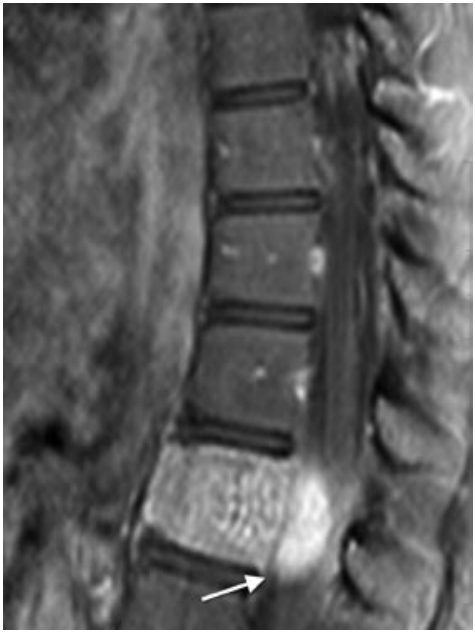

C. Sagittal MRI T1 sequence post IV contrast medium shows an expansile T12 vertebral body with diffuse enhancement and an enhancing posterior epidural soft tissue mass (arrow) severely compressing the thecal sac.

Fig. 3. Sagittal MRI T1 sequence of expansile T12 vertebral body with diffuse hypointensity and a posterior epidural hypointense soft tissue mass (arrow) severely compressing the thecal sac.

\section{Discussion}

The prevalence of back pain in the pediatric age group is less as compared to adults. Masiero et al (5) reported an increased incidence in teenagers, females, and sedentary children, and children with a family history of low back pain. The lifetime prevalence of low back pain in children and teenagers varies between $3 \%$ and $63 \%$ (5). When children or adolescents seek medical care for back pain, it is highly likely that an underlying pathology will be identified. Common causes of back pain include nonspecific pain or muscle strain, herniated disc, spondylolysis, scoliosis, and Scheuermann's kyphosis. Less common causes include tumor, infection, and sickle cell crisis (6), though obesity is a cause which is rapidly becoming more important due to myriad comorbid conditions and altered biomechanical forces (7). It is generally accepted that the following factors warrant immediate evaluation: patient age younger than 4 years, persistent symptoms, selfimposed activity limitations, systemic symptoms, increasing discomfort, persistent nighttime pain, and neurologic symptoms (6).

We present an unusual cause of back pain with an aggressive hemangioma causing severe spinal cord compression. This is an unusual pathology and only 3 cases in children less than 17 year old have been reported in the literature (1-3). We are presenting, to the best of our knowledge, the first case demonstrating the evolution of an aggressive vertebral hemangioma demonstrating stability for a period followed by increased growth with subsequent compression of the spinal cord. The other 3 cases 
described presented with acute compression of the spinal cord. This case raises important questions related to the management of this clinical condition. How can we differentiate between non-aggressive and aggressive hemangioma, between aggressive hemangioma and other pathological diagnoses, and when should we pursue a conservative versus surgical or embolization therapeutic approach? There are multiple case reports of hemangioma increasing in size during pregnancy due to the mass effect on the vertebral venous plexus by gravid uterus, and the effects of progesterone and estrogen (8). There is an inverse relationship between the amount of intraosseous fatty stroma and aggressiveness of the lesion (9). Asymptomatic vertebral hemangioma classically shows high signal intensity on both T1 and T2 sequences due to its fat content. Also, the symptomatic vertebral hemangiomas are extremely hyperintense on the $\mathrm{T} 2$ sequence that helps to distinguish them from lymphoma and other highly cellular neoplasms (10). Involvement of the posterior elements and the whole vertebral body is more in keeping with symptomatic vertebral hemangioma with less marked pathological compression of the whole vertebral body as compared to metastatic disease. Freidman (10) suggested that aggressive hemangioma should be considered with the following characteristics: female patient, marked hyperintensity on T2, intrasseous signal voids or mottled appearance, midthoracic location, and enlarged paraspinal vessels. Differential diagnoses would include osseous tumor such as Ewing sarcoma (11) or Hemangioblastoma (12).

Different therapies discussed in the literature include embolization and ethanol injection, surgical resection, methacrylate vertebroplasty, and radiation $(13,14)$. Vertebroplasty with the intraspinal component may lead to worsening of compression by filling the intraspinal hemangioma component (13). Radiotherapy may lead to cord necrosis and acute cord compression may not benefit because of the delayed effect (15). Embolization and ethanol injection have some complications such as spinal cord infarct, leakage of injected material into the subarachnoid space, ethanol induced osteonecrosis, Brown-sequard syndrome and paravertebral abscess (14). Surgical treatment is usually reserved for cases involving spinal cord compression with progressive neurological deficits.

\section{Conclusion}

We present a longitudinal study of vertebral hemangioma in a pediatric female patient, which grew aggressively after a period of stability over a period of 20 months and resulted in an epidural mass and cord compression. We describe the imaging features and discuss differentiating features between the asymptomatic hemangioma and malignant lesions. In our patient, among the risk factors described in the literature for an aggressive hemangioma, female gender and involvement of posterior elements were positive. We recommend a close follow-up of cases diagnosed with asymptomatic vertebral hemangioma in children particularly if there are any risk factors for the development of aggressive vertebral hemangioma. MRI would be a more suitable imaging modality to follow them with due to its excellent soft tissue contrast and lack of ionizing radiation. Management of a female patient in the pediatric age group with asymptomatic vertebral hemangioma will be more challenging. Here, the decision would be between prophylactic management before any changes occur related to potential risk factors of pregnancy and hormonal changes at a later age versus watchful surveillance. However, therapy has its attendant risks as well and the natural history of this lesion is not yet well described in children. We need to study aggressive vertebral hemangioma further especially in children to understand the natural course and help identify lesions which have aggressive potential and prevent spinal cord compression. This will also help to manage the lesion earlier with less aggressive therapies such as embolization and ethanol injection rather than surgery. 


\section{References}

1. Hadjipavlou A, Tosounidis T, Gaitanis I, Kakavelakis K, Katonis P. Balloon kyphoplasty as a single or as an adjunct procedure for the management of symptomatic vertebral haemangiomas. J Bone Joint Surg Br 2007; 89:495-502.

2. Duprez T, Lokietek W, Clapuyt P, DeMerlier Y, Malghem J, Gadisseux JF. Multiple aggressive vertebral haemangiomas in an adolescent: A case report. Pediatr Radiol 1998; 28:51-53.

3. Cross JJ, Antoun NM, Laing RJC, Xuereb J. Imaging of compressive vertebral haemangiomas. Eur. Radiol 2000; 10:9971002.

4. Hernalsteen D, Cosnard G, Duprez T. Long term (6 years) follow up of untreated multiple aggressive vertebral hemangiomas in an adolescent. Pediatric Radiology 2004; 34:831-832.

5. Masiero S, Carraro E, Celia A, Sarto D, Ermani $M$. Prevalence of nonspecific low back pain in schoolchildren aged between 13 and 15 years.: Acta Pædiatrica 2008; 97:212-216.
6. Bernstein RM, Cozen H. Evaluation of back pain in children and adolescents. American Family Physician 2007; 76:1669-1676.

7. Choudhary AK, Donnelly LF, Racadio JM, Strife J. Diseases associated with childhood obesity. AJR 2007; 188:1118-1130.

8. Yuksel M, Yuksel KZ, Tuncel D, Zencirci B, Bakaris S. Symptomatic vertebral hemangioma related to pregnancy: Emerg Radiol 2007; 13:259-263.

9. Laredo JD, Assouline E, Gelbert F, Wybier M, Merland JJ, Tubiana JM. Vertebral hemangiomas: Fat content as a sign of aggressiveness. Radiology JM 1990; 177:467-472.

10. Friedman DP. Symptomatic vertebral hemangiomas: MR findings. AJR 1996; 167:359-364.

11. Bemporad JA, Sze G, Chaloupka JC, Duncan C. Pseudohemangioma of the vertebra: an unusual radiographic manifestation of primary ewing's sarcoma. AJNR Am J Neuroradiol 1999; 20:1809-1813.
12. Steinmetz MP, Claybrooks R, Krishnaney A, Prayson RA, Benzel EC. Surgical management of osseous Hemangioblastoma of the thoracic spine: Technical case report. Neurosurgery 2005; 57:E405; discussion E405.

13. Doppman JL, Oldfield EH, Heiss JD.Symptomatic vertebral hemangiomas: Treatment by means of direct intralesional injection of ethanol. Radiology 2000; 214:341-348.

14. Chen HI, Heuer GG, Zaghloul K, Simon SL, Weigele JB, Grady MS. Case report. Lumbar vertebral hemangioma presenting with the acute onset of neurological symptoms. J Neurosurg Spine 2007; 7:80-85.

15. Fox MW, Onofrio BM. The natural history and management of symptomatic and asymptomatic vertebral hemangiomas. J Neurosurg 1993; 78:36-45. 